\title{
Central nervous system inflammatory demyelinating diseases with stroke-like onset and their responses to thrombolysis
}

\author{
Zhi Zhou ${ }^{1} \cdot$ Duan Qian ${ }^{1} \cdot$ Lei Liu $^{2} \cdot$ Weihe Zhang ${ }^{2} \cdot$ Zunjing Liu $^{2}$
}

Received: 1 April 2015/ Accepted: 13 June 2015/Published online: 24 June 2015

(C) The Author(s) 2015. This article is published with open access at Springerlink.com

Keywords Demyelinating diseases $\cdot$ Stroke mimics . Thrombolysis · Baló's concentric sclerosis · Neuromyelitis optica spectrum disorder

\section{Dear Editor,}

Sometimes, given the similarity of initial symptoms, diagnostic confusion may arise between demyelinating disease and stroke. Misdiagnosis of MS likely occurs more frequently and has been documented well [1, 2]. However, as far as we know, demyelinating diseases treated with thrombolysis have never been reported before. We present two inflammatory demyelinating cases with stroke-like onset which were treated with thrombolysis.

\section{Case reports}

\section{Case 1}

\section{Presentation}

A 28-year-old woman was admitted with right-side weakness for $50 \mathrm{~min}$. Neurological examination showed right nasolabial fold flattening and 1 of 5 strength in the right limbs. Initial head CT revealed low density on the left

\section{Zunjing Liu}

liuzunjing@163.com

1 Cadre Ward, China-Japan Friendship Hospital, Beijing, China

2 Department of Neurology, China-Japan Friendship Hospital, Beijing, China periventricular area. Cerebral MRI scan showed a long T1 and $\mathrm{T} 2$ signal intensity lesion on the left periventricular area, which had restricted diffusion on diffusion weighted imaging (DWI) (Fig. 1). She was diagnosed as acute ischemic stroke and treated with intravenous thrombolysis $4 \mathrm{~h}$ after onset.

She improved gradually. Neurological examination only showed hemiparesis of the right $16 \mathrm{~h}$ later. Her past history was not remarkable.

\section{Hospital course}

Results of blood work including biochemical, immunologic and infectious assessment were normal except for mild elevated triglycerides. Magnetic resonance angiography (MRA) was normal.

However, her strength worsened 5 days after thrombolysis. Neurologic examination showed right central facial palsy and 2 of 5 strength in the right limbs. With a deteriorating clinical status, we suspected the diagnose of stroke. Lumbar puncture revealed a clear fluid liquid with normal cellularity and mild elevated protein. AQP 4-antibody (AQP4-Ab) was negative in blood and CSF. Contrastenhanced MRI showed a gadolinium (Gd) enhancement lesion on the left posterior lateral ventricle angle which had a long T1 and T2 signal intensity (Fig. 2). The lesion showed a defined ring structure suggestive of Baló's concentric sclerosis (BCS). After this finding, the diagnosis was revised to BCS. Other investigations were performed to support the diagnosis. Her cervical and thoracic spine MRI were normal.

Intravenous of methylprednisolone ( $1 \mathrm{~g} /$ day for 5 days) was initiated followed by oral treatment. She gradually recovered with 4 of 5 strength in the right 5 days later. 

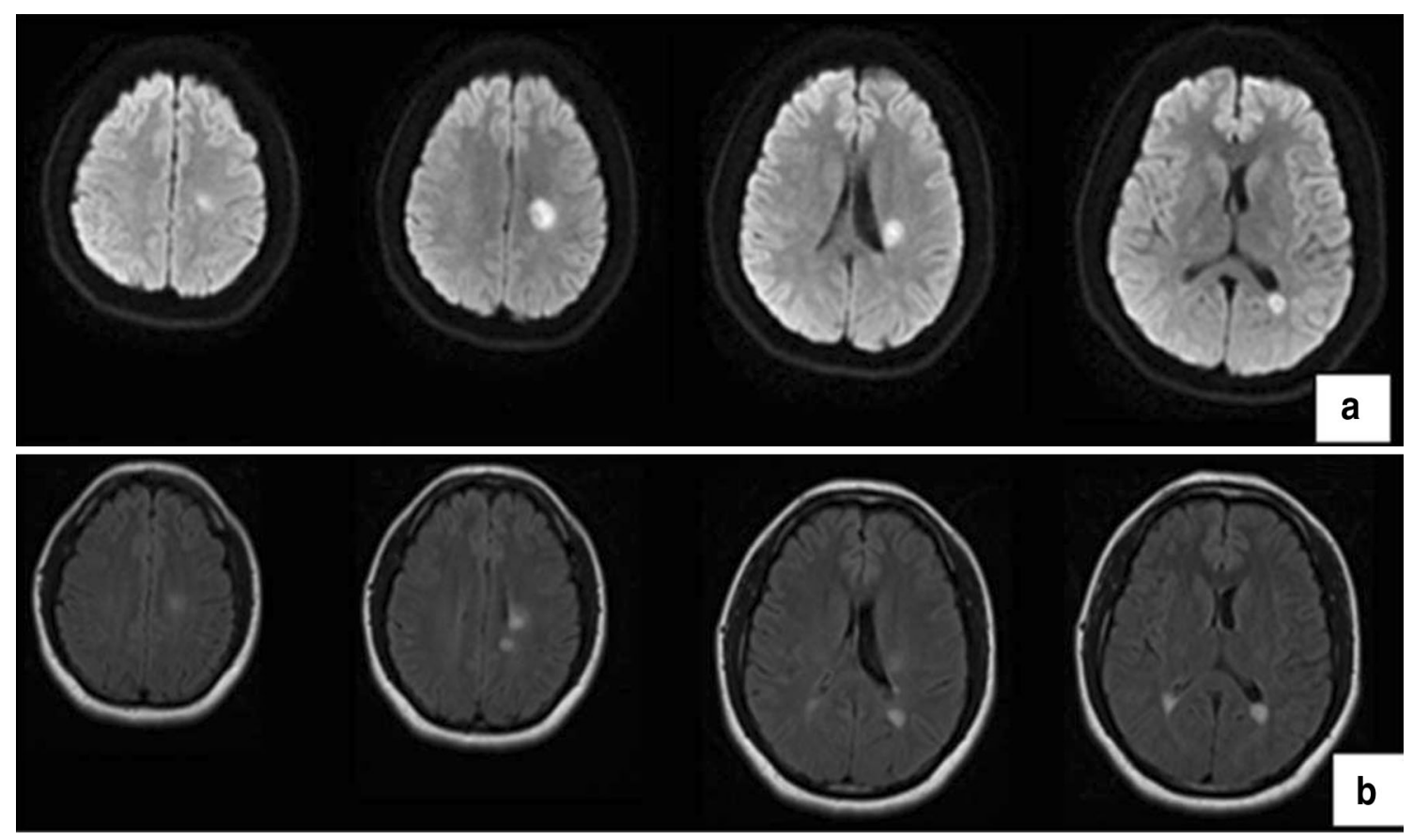

Fig. 1 Brain MRI of case 1 showed a long T2 (a) signal intensity lesion on the left periventricular area, which had restricted diffusion on DWI (b)
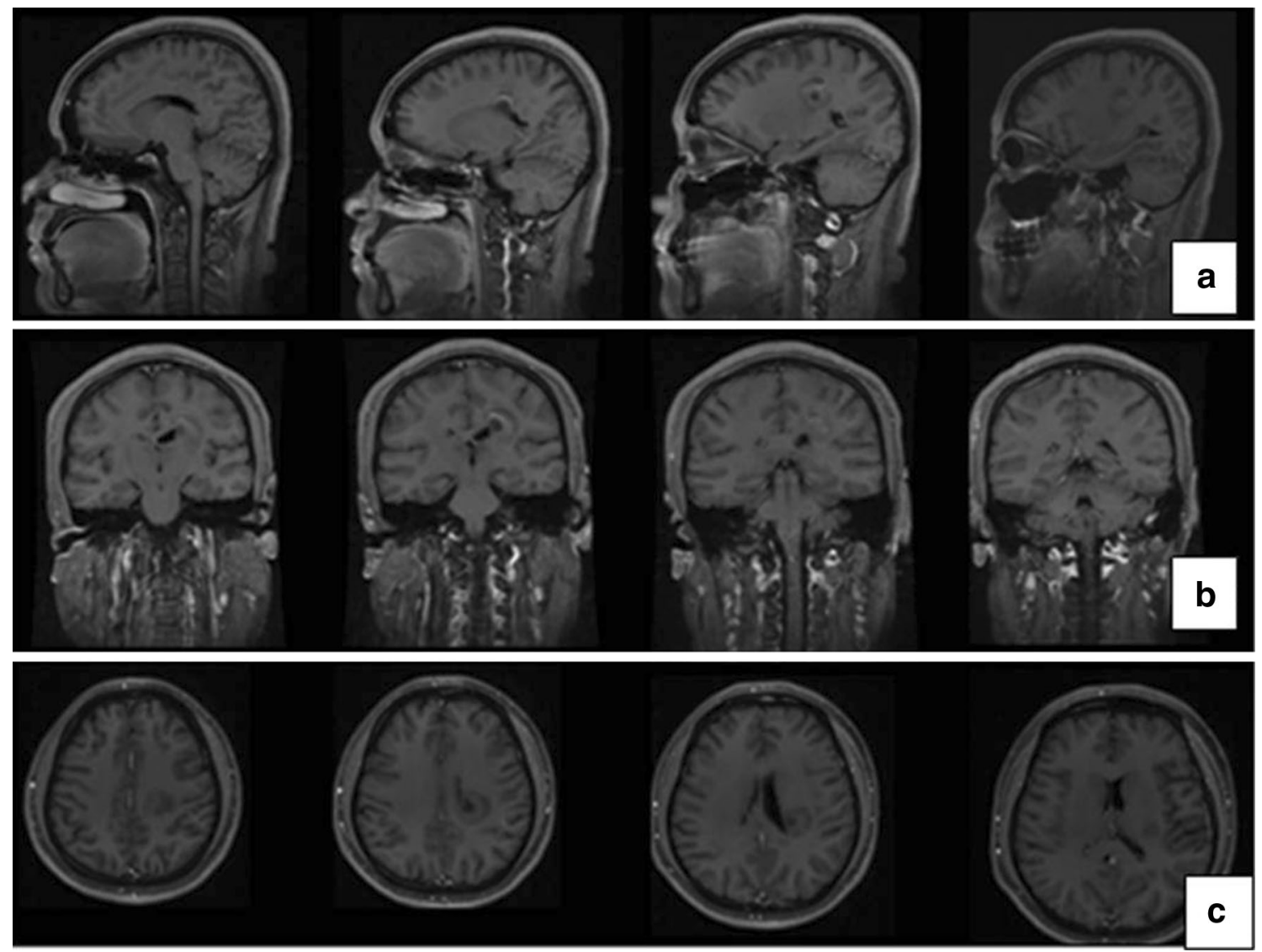

Fig. 2 Contrast-enhanced MRI of case 1 showed a Gd enhancement lesion on the left posterior lateral ventricle angle which had long T1 signal intensity 


\section{Case 2}

\section{Presentation}

A 56-year-old woman began to hiccup and vomit frequently 3 months ago, which progressively worsen over 1 month, combined with dysphagia and hoarseness. Brain MRI of local hospital revealed long signal intensity on T1 and T2 weighted imaging involving the dorsolateral medullary, which had restricted diffusion on DWI, and MRA was unremarkable (Fig. 3). She was diagnosed as stroke and treated with aspirin. 30 days after onset, she suddenly deteriorated with flaccid paralysis of the right limbs, with 1 of 5 strength. Cerebral arterial angiography was performed in local hospital and she was treated with intra-arterial urokinase in right vertebral artery. Her condition improved 3 days after thrombolysis and she could walk by herself 10 days later.

One month later, she abruptly developed left hemiparesis. Brain and cervical MRI revealed long signal intensity lesion on T1 and T2-weighted imaging involving the hypothalamic, periaqueductal, medulla and cervical medullary (Fig. 4).

She had an upper respiratory tract infection 1 week prior to onset. Her past history was not remarkable except for well-controlled hypertension.

\section{Hospital course}

Cranial nerve examination was remarkable for bilateral torsional nystagmus, left nasolabial fold flattening and decreased sensation in the left. Motor examination revealed 1 of 5 in the left limbs. Blood work were normal. CSF electrophoresis demonstrated oligoclonal bands with a high IgG index. AQP4-Ab was positive (1:10) both in blood and CSF. Thoracic spine MRI studies did not reveal any abnormality. A diagnosis of neuromyelitis optica spectrum disorder was made based on Wingerchuk's criteria (myelitis associated with brain lesions typical of neuromyelitis optica).

Intravenous of methylprednisolone ( $1 \mathrm{~g} /$ day) was initiated and then converted to oral after 5 days. Azathioprine was then added. She gradually recovered 3 days after treatment and could walk by herself 20 days later.

\section{Follow-up}

Cerebral MRI performed 7 months later did not show any new lesions, and the dorsolateral medullary lesion had significantly reduced in size (Fig. 5). She has not experienced any clinical relapses during the follow-up period.

\section{Discussion}

The necessity for rapid thrombolysis in acute ischemic stroke may lead to treatment of patients with conditions mimicking stroke. It is remarkable that both of the two patients got relieved in a short time after thrombolysis. The patient in case 1 improving in $4.5 \mathrm{~h}$ made diagnosis more difficult. It needs to be questioned whether the patient of case 2 should receive intra-arterial thrombolysis according to the indications [3], whatever, she recovered in short time.

Recent studies suggest that fibrinogen plays an important role not only in coagulation but also in inflammation [4]. Some authors reported that thrombin activity preceded onset of neurological signs, increased at disease peak, and correlated with fibrin deposition, microglial activation, demyelination, axonal damage, and clinical severity [4]. The role of coagulation-fibrinolysis system in experimental autoimmune encephalomyelitis (EAE) was confirmed using batroxobin, the thrombin inhibitor. They reported that batroxobin treatment
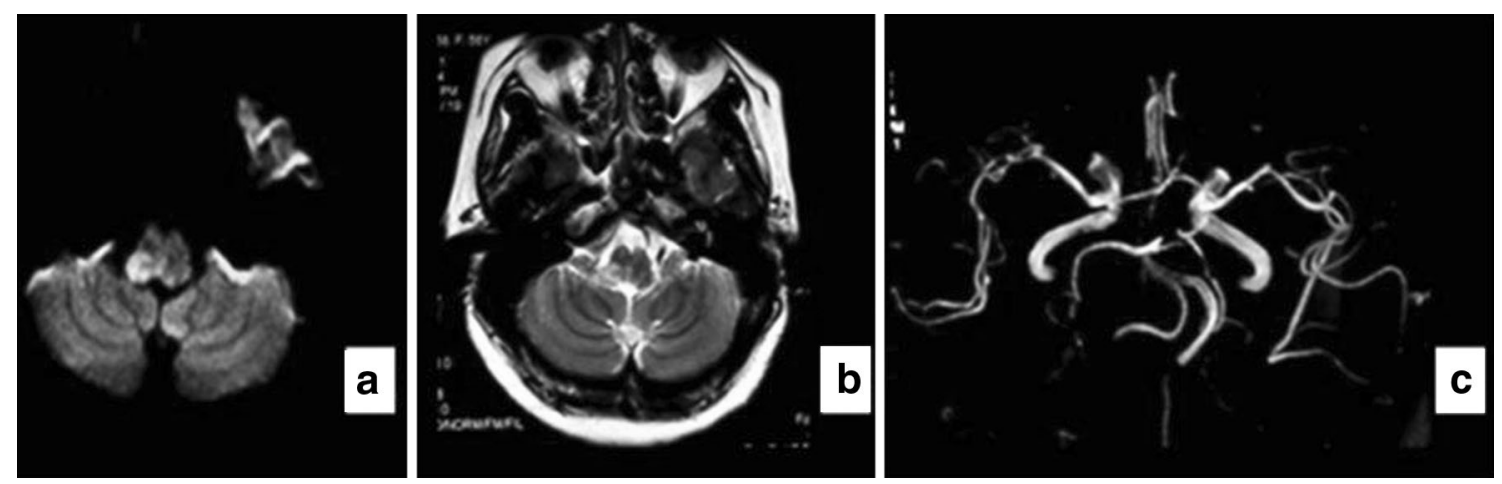

Fig. 3 Brain MRI of local hospital in case 2 revealed long signal intensity involving the dorsolateral medullary on T2 weighted imaging (a), which had restricted diffusion on DWI (b), and her MRA was unremarkable (c) 

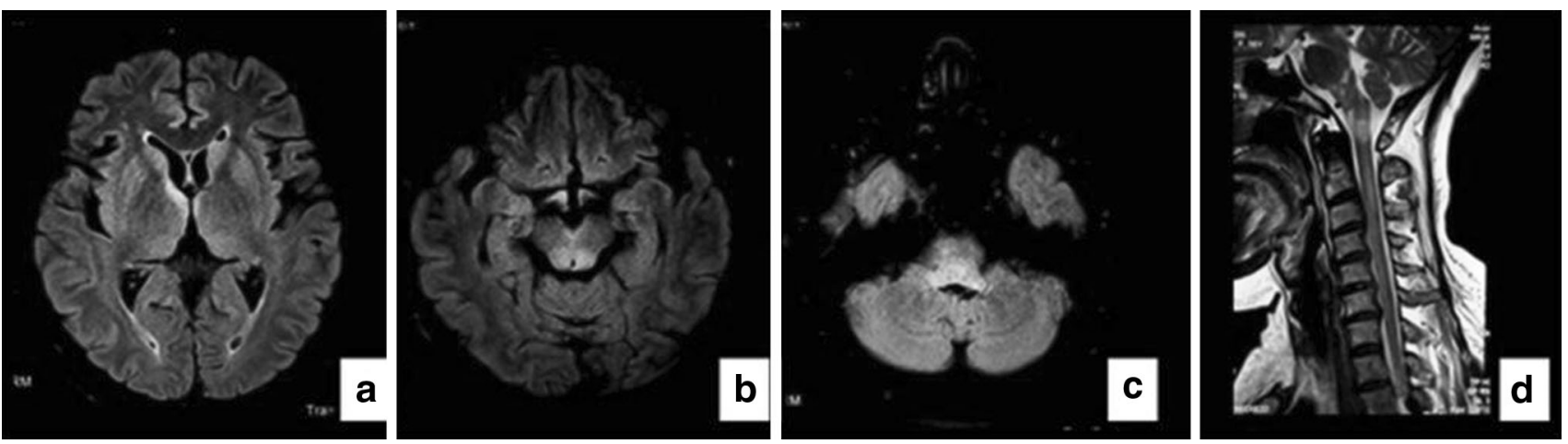

Fig. 4 Brain and cervical MRI of case 2 revealed long signal intensity lesion on T2 weighted imaging involving the hypothalamic (a), periaqueductal (b), medulla (c) and cervical medullary (d)
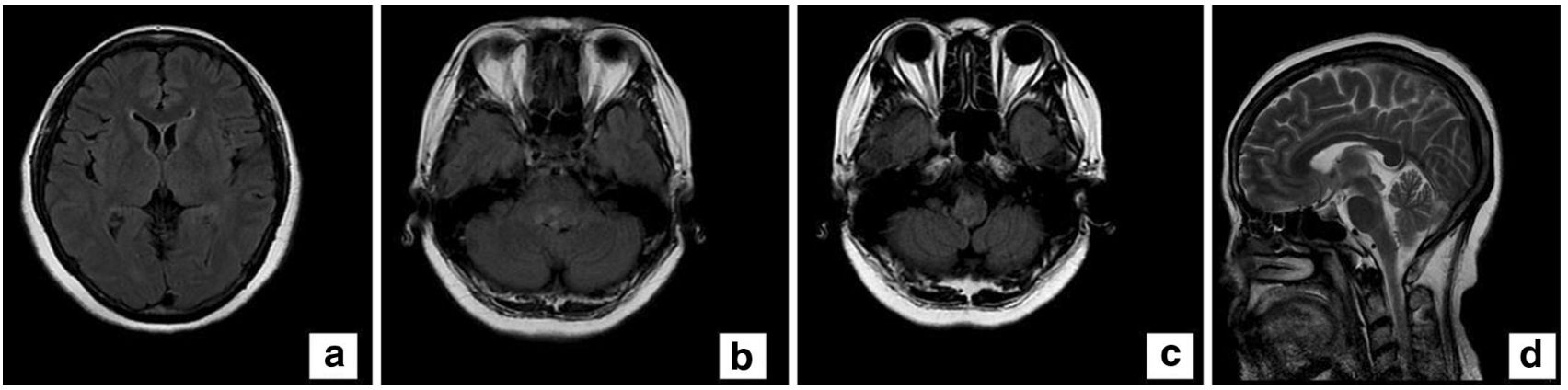

Fig. 5 Brain MRI of case 2 in the follow-up did not show any new lesions, and the dorsolateral medullary lesion had significantly reduced in size

suppressed clinical signs of cell transferred EAE, and deposition of fibrin around the vessels in the spinal cord was markedly suppressed in batroxobin-treated rats. These findings suggest that batroxobin suppresses EAE by preventing fibrin deposition, and provide evidence that CNSassociated deposition of fibrin and ensuing fibrinolysis, together with increased permeability of blood brain barrier (BBB), are related prerequisites for the clinical manifestation of EAE [5]. Using mice deficient in tissuetype plasminogen activator (tPA-/-), East et al. investigated the involvement of the PA system on the clinical and pathological features of EAE. They found that tPA-I- mice suffered an early and a more severe acute disease characterized by incomplete recovery when compared to wild-type controls [6]. A better understanding of the mechanism of inhibition of coagulation-fibrinolysis system facilitate disease pathogenesis may present a new strategy for pharmacological intervention in inflammatory demyelinating diseases.

Acknowledgment This article is supported by China-Japan Friendship Hospital Youth Science and Technology Excellence Project (2014-QNYC-A-04).
Compliance with ethical standards

Conflict of interest There are no conflicts of interest to declare.

Open Access This article is distributed under the terms of the Creative Commons Attribution 4.0 International License (http://creativecommons.org/licenses/by/4.0/), which permits unrestricted use, distribution, and reproduction in any medium, provided you give appropriate credit to the original author(s) and the source, provide a link to the Creative Commons license, and indicate if changes were made.

\section{References}

1. Rosso C, Remy P, Creange A, Brugieres P, Cesaro P et al (2006) Diffusion-weighted MR imaging characteristics of an acute strokelike form of multiple sclerosis. AJNR Am J Neuroradiol 27:1006-1008

2. Przeklasa-Auth M, Ovbiagele B, Yim C, Shewmon DA (2010) Multiple sclerosis with initial stroke-like clinicoradiologic features: case report and literature review. J Child Neurol 25:732-737

3. Huang YH, Zhuo ST, Chen YF, Li MM, Lin YY et al (2013) Factors influencing clinical outcomes of acute ischemic stroke treated with intravenous recombinant tissue plasminogen activator. Chin Med J (Engl) 126:4685-4690 
4. Davalos D, Akassoglou K (2012) Fibrinogen as a key regulator of inflammation in disease. Semin Immunopathol 34:43-62

5. Inoue A, Koh CS, Shimada K, Yanagisawa N, Yoshimura K (1996) Suppression of cell-transferred experimental autoimmune encephalomyelitis in defibrinated Lewis rats. J Neuroimmunol 71:131-137
6. East E, Baker D, Pryce G, Lijnen HR, Cuzner ML et al (2005) A role for the plasminogen activator system in inflammation and neurodegeneration in the central nervous system during experimental allergic encephalomyelitis. Am J Pathol 167:545-554 